\title{
Euphorbia humifusa Willd exerts inhibition of breast cancer cell invasion and metastasis through inhibition of TNFa- induced MMP-9 expression
}

Soon Young Shin ${ }^{1,2}$, Chang Gun Kim², You Jung Jung ${ }^{2}$, Yearam Jung ${ }^{3}$, Hyeryoung Jung ${ }^{3}$, Jihyun Im $^{3}$, Yoongho Lim ${ }^{3,4^{*}}$ and Young Han Lee ${ }^{1,2,4^{*}}$ (D)

\begin{abstract}
Background: Breast cancer is the most common type of malignancy in women worldwide. Euphorbia humifusa Willd (EuH) is a plant that is widely used as a traditional medicine. However, no systemic studies on the anti-cancer effects of EuH have been reported. The aim of this study is to evaluate the anti-metastatic effect of the EuH.

Methods: Ethyl acetate fraction was prepared from EuH methanol extracts (EA/EuH). Inhibitory effect of EA/EuH on cell migration was determined using an in vitro scratch-wound healing assay. The anti-invasive activity was determined by in vitro three-dimensional spheroid culture system and in vivo syngenic experimental lung metastasis experiment. Gene expression profiles were analyzed by using RT-PCR, real-time PCR, and luciferase reporter assay systems.

Results: Ethyl acetate fraction from the EuH extract (EA/EuH) inhibited the migration and invasive capabilities of highly metastatic MDA-MB-231 breast cancer cells and attenuated syngeneic lung metastasis of mouse 4 T1 breast cancer cells in vivo. Mechanistically, EA/EuH decreased tumor necrosis factor alpha (TNFa)-induced matrix metalloproteinase (MMP)-9 mRNA expression through the inhibition of NF-kB activity in MDA-MB-231 cells.
\end{abstract}

Conclusion: EuH may be beneficial in the prevention of invasion and metastasis of early stage breast cancer and can be served as an anti-metastatic agent or adjuvant therapy against metastatic breast cancer.

Keywords: Euphorbia humifusa Willd, Invasion, Metastasis, Matrix metalloproteinase-9, Tumor necrosis factor alpha, Nuclear factor-kappa B

\section{Background}

Breast cancer is the most common type of malignancy in women worldwide, accounting for approximately $23 \%$ of total cancer cases and $14 \%$ of total cancerrelated deaths [1]. Breast cancer cells often exhibit high invasive and metastatic properties. Metastasis is the uncontrolled spread of primary tumor cells to other sites in the body and commonly occurs in the late stages of cancer. Like other cancer types, the prognosis of breast

\footnotetext{
* Correspondence: yoongho@konkuk.ac.kr; yhlee58@konkuk.ac.kr

${ }^{3}$ Division of Bioscience and Biotechnology, College of Biological Science and

Biotechnology, BMIC, Konkuk University, Seoul, Republic of Korea

'Department of Biological Sciences, College of Biological Science and

Biotechnology, Konkuk University, Seoul, Republic of Korea

Full list of author information is available at the end of the article
}

cancer patients is strongly influenced by the stage of metastasis.

The tumor microenvironment is the cellular environment that surrounds the tumor site, and is composed of extracellular matrix (ECM), blood vessels, and various cell types, including stromal fibroblasts and infiltrative immune cells. Tumor necrosis factor alpha (TNFo) is a pro-inflammatory cytokine involved in the modulation of systemic inflammation. In the tumor microenvironment, TNF $\alpha$ is produced by tumor cells as well as tumor-associated cells and plays a central role in promoting tumor invasion and metastasis [2]. During malignant progression, breast epithelial cells undergo a transition to mesenchymal-type cells (epithelial-to- 
mesenchymal transition). These cells are migratory and can invade through the surrounding ECM. Matrix metalloproteinases (MMPs) are zinc-dependent proteases that are mainly involved in tissue remodelling via various physiological and pathological processes that degrade ECM proteins. Emerging evidence has emphasized the role of MMPs in migration and invasion of cancer cells through the breakdown of ECM and basement membranes [3].

The transcription factor NF- $\mathrm{KB}$ regulates many genes in control of inflammatory responses, cell proliferation, cell survival, angiogenesis, and metastasis [4]. The NF$\kappa B$ family consists of five members, including NF- $\kappa B 1$ (also called p50), NF- $\mathrm{kB} 2$ (also called p52), RelA (also called p65), RelB, and c-Rel [5]. In unstimulated cells, $\mathrm{NF}-\mathrm{\kappa B}$ is sequestered in the cytoplasm by the inhibitory protein $I_{\kappa} B$, which hinders the nuclear localization of $\mathrm{NF}-\kappa \mathrm{B}$. Upon stimulation of the cells, IкB kinases (IKK) stimulate NF-kB through the phosphorylation of ІкB. This results in a degradation and dissociation of $І \textrm{B}$ from NF- $\mathrm{kB}$ leading to the translocation of NF- $\mathrm{kB}$ into the nucleus. In many different types of human cancers, a constitutively activated NF- $\mathrm{KB}$ is common [6]. Aberrant activation of NF- $\mathrm{KB}$ is known to be associated with the progression of breast cancer [7], particularly the promotion of tumor cell invasion, migration, and metastasis through the upregulation of MMP-9 expression [8]. Therefore, it seems likely that the inhibition of NF- $\mathrm{KB}$ activity is required for the prevention and treatment of cancer. It should be noted that several agents inhibiting $\mathrm{NF}-\mathrm{kB}$ functions are currently in clinical use or undergoing clinical development as cancer chemotherapeutics $[4,9-11]$.

Euphorbia humifusa Willd (EuH), known as TtangBin-Dae in Korea or Di-Jin-Cao in China, is a dicotyledonous, polypetalous plant widely distributed in eastern Asia. In Korea, EuH has been used as a traditional medicinal plant for the treatment of diarrhoea, jaundice, dysentery, enteritis, diabetes, and asthma [12, 13]. Previous phytochemical studies have isolated multiple bioactive compounds from the EuH, including euphorbinoside dehydropicrorhiza acid methyl diester, flavone glucosides, apigenin glycosides, tannins, $\alpha$-pyrrolidinonoids, lanostane triterpenoids [14-16], exhibiting various pharmacological actions, including antifungal, anti-inflammatory, vasorelaxant, and anti-viral properties [12, 16-18].

However, the effects of EuH on cancer progression, such as invasion and metastasis, have not been elucidated. In this study, we examined the anti-metastatic effects of $\mathrm{EuH}$ on the highly metastatic human breast cancer cell line MDA-MB-231. Our results indicate that $\mathrm{EuH}$ inhibits migration, invasion, and experimental metastasis of MDA-MB-231 cells. Furthermore, EuH reduces TNFo-induced MMP-9 expression through the inhibition of NF- $\mathrm{kB}$ activity. The data suggest that EuH may be beneficial in the prevention of an invasion and metastasis of early stage breast cancer.

\section{Methods \\ Cells and chemicals}

Human breast carcinoma cells (MDA-MB-231, MDAMB-435, T47D, and MCF7) were obtained from the American Type Culture Collection (ATCC, Manassas, VA, MD, USA). Mouse mammary carcinoma cells (4 T1) were kindly provided by Dr. Jeong-Seok Nam (Gwangju Institute of Science and Technology, Gwangju, Korea). Cells were grown in Dulbecco's modified Eagle's medium (Corning Cellgro, Manassas, VA, USA) supplemented with $10 \%(\mathrm{v} / \mathrm{v})$ heat-inactivated foetal bovine serum (Corning Cellgro). TNF $\alpha$, methanol, n-hexane, chloroform, ethyl acetate, n-butanol, dimethylsulfoxide (DMSO), Tris-base, HEPES, Triton X-100, glycerol, leupeptin, phenylmethylsulfonyl fluoride (PMSF) were purchased from Sigma-Aldrich (Saint Louis, MO, USA). SDS, acrylamide, bisacrylamide, ammoniuk persulfate, nitrocellular membranes were from Bio-Rad Laboratories (Hercules, CA, USA). Pierce ${ }^{\mathrm{Th}}$ BCA Protein Assay Reagent was obtained from Thermo Scientific (Rockford, IL, USA).

\section{Preparation of the E. humifusa Willd extracts}

Air dried E. humifusa Willd (EuH) was purchased from an herbal market from Gyungdong Pharmaceutical Market (Seoul, Republic of Korea) and taxonomically identified by a galenical pharmacist, Dr. Hi Jae Cho (College of Biological Science and Biotechnology, Konkuk University, Seoul, Republic of Korea). A voucher specimen was deposited at the College of Biological Science and Technology, Konkuk University, Korea. EuH $(3 \mathrm{~kg})$ was soaked in methanol for 3 days. The methanolic extract (130 g) was obtained under reduced pressure using a rotary evaporator. By polarity based fractionation, five fractions were collected from $35.93 \mathrm{~g}$ of methanol extract: n-hexane (10.08 g; $28.1 \%)$, chloroform (1.36 g; $3.8 \%)$, ethyl acetate (4.91 g; $13.7 \%)$, n-butanol (8.48 g; $23.6 \%)$, and aqueous fraction (11.12 g; $30.9 \%$ ). Each fraction was dried using a freeze-dryer and dissolved in DMSO at $10 \mathrm{mg} / \mathrm{mL}$.

\section{Cell migration assay}

Migration of MDA-MB-231 cells was determined using an in vitro scratch-wound healing assay, as described previously [19]. Migrated cells were photographed with an EVOS $^{\circ}$ FL Auto Cell Imaging System (Life Technologies, Carlsbad, CA, USA). Cells migrated into the gap area in (A) were quantified in a field of view using ImageJ software (https://imagej.nih.gov/ij/; Center for Information Technology, National Institute of Health, Bethesda, MA, USA). 


\section{Actin reorganization}

Polymerized F-actin was examined using the Rhodaminephalloidin-based F-Actin Visualization Biochem Kit (Cytoskeleton, Inc.; Denver, CO, USA), according to the manufacturer's instructions. Images were captured using an EVOS FL Auto Cell Imaging System (Life Technologies).

\section{Three-dimensional spheroid culture and invasion assay}

Three-dimensional invasion assay was performed using a Cultrex 3-D Spheroid Cell Invasion Assay kit (Trevigen, Inc., Gaithersburg, MD, USA), as described previously [20]. Invasive protrusions into ECM were visualized an EVOS FL Auto Cell Imaging System.

\section{Syngenic experimental lung metastasis assay}

Six-week-old female Balb/c mice were purchased from YoungBio (Seongnam, Gyeonggi-do, Korea). All animal experiments were conducted following the standards and procedures approved by the Konkuk University Institutional Animal Care and Use Committee (No.KU15194). 4. T1 mammary carcinoma cells $\left(6 \times 10^{4}\right.$ cells $\left./ 50 \mu \mathrm{L}\right)$ were injected into the spleen as described previously [21]. One-day after intra-splenic implantation of tumor cells, PBS $(n=5)$ or EA/EuH $(10 \mathrm{mg} / \mathrm{kg} ; n=7)$ was administered daily intraperitoneally. Mice were sacrificed after 8 days of cell inoculation, and lung tissues were stained by routine hematoxylin and eosin (H\&E).

\section{RT-PCR and quantitative real-time PCR}

Total RNA was extracted using Isol-RNA lysis reagent (NucleoZOL; Clontech, Mountain View, CA, USA), and the synthesis of cDNA was carried out using an iScript cDNA synthesis kit (Bio-Rad, Hercules, CA, USA). RTPCR was performed as described previously [22]. For quantitative real-time PCR, TaqMan-iQ supermix kit (Bio-Rad) was used with the Bio-Rad iCycler iQ thermal cycler according to the manufacturer's instruction. The TaqMan fluorogenic probes and gene-specific PCR primers for MMP-9 and glyceraldehyde-3-phosphate dehydrogenase (GAPDH) were described previously [22]. The relative fold changes were normalized to GAPDH mRNA in the same sample.

\section{NF-KB-dependent transcriptional activity assay}

MDA-MB-231 cells were transfected with $0.1 \mu \mathrm{g}$ of the $5 \times N F \kappa B-L u c$ plasmid, containing five repeats of NF-kB binding sites and treated with $10 \mathrm{ng} / \mathrm{mL}$ TNF $\alpha$ in the absence and presence of $\mathrm{EA} / \mathrm{EuH}$, as described previously [20]. The luciferase activities were measured with a Centro LB960 luminometer (Berthold Technologies; Bad Wildbad, Germany).

\section{Immunoblot analysis}

Cells were lysed in $20 \mathrm{mM}$ HEPES (pH 7.2), $1 \%$ (v/v) Triton X-100, $10 \%$ (v/v) glycerol, $150 \mathrm{mM} \mathrm{NaCl}$, $10 \mu \mathrm{g} / \mathrm{mL}$ leupeptin, and $1 \mathrm{mM}$ PMSF. Protein extracts $(20 \mu \mathrm{g}$ per sample) were separated via $10 \%$ SDS-PAGE, transferred to nitrocellulose membranes, and incubated with appropriate primary and secondary antibodies. Primary antibodies against phosphoIкB (Ser32) and phospho-RelA/p65 (Ser536) were obtained from Cell Signaling Technology (Beverly, MA, USA), and an antibody against GAPDH was from Santa Cruz Biotechnology (Dallas, TX, USA). The blots were developed using an enhanced chemiluminescence detection system (GE Healthcare, Piscataway, NJ, USA). The relative protein band intensities were determined using ImageJ software.

\section{Immunofluorescence microscopy}

MDA-MB-231 cells plated on coverslips were treated with or without $10 \mathrm{ng} / \mathrm{mL} \mathrm{TNF} \alpha$ in the absence and presence of EA/EuH $(5 \mu \mathrm{g} / \mathrm{mL})$ for $30 \mathrm{~min}$, followed by fixation, permeabilization, and incubation of primary antibodies specific to $\alpha$-tubulin and phospho-p65/RelA (Ser536) for $2 \mathrm{~h}$, as described previously [23]. Fluorescent staining cells were examined under an EVOS FL fluorescence microscope (Advanced Microscopy Group; Bothell, WA, USA).

\section{MMP-9 promoter reporter assay}

Construction of the MMP-9 promoter reporters, wildtype pMMP9-Luc $(-925 /+13)$ and disrupted NF-kB binding site pMMP9-Luc(-925/+13)mtNFkB, was described previously [24]. Transfection of promoter reporters and luciferase reporter assay were described previously [24]. Luminescence was measured using a Centro LB960 dual luminometer (Berthold Technologies).

\section{Statistical analysis}

All experiments were performed in triplicate. Data are presented as the mean \pm SD. Statistical analysis was performed by one-way ANOVA followed by Sidak's multiple comparisons test using GraphPad Prism version 7.0 software (GraphPad Software Inc., La Jolla, CA). P-values < 0.05 were considered statistically significant.

\section{Results and discussion}

Ethyl acetate fraction of EuH (EA/EuH) inhibits TNFainduced motility of MDA-MB-231 breast cancer cells

$\mathrm{NF}-\mathrm{kB}$ is a transcription factor found in almost all animal cells and plays a key role in regulating cellular responses, including immune responses, cell proliferation, and survival in diverse cell types [25]. Aberrant activation of NF- $\mathrm{KB}$ has been implicated in the progression of breast cancer [7]. Among the NF-kB family, 
RelA/p65 and p50 complex is the most common form studied intensively in human cells. Upon stimulation, I $\mathrm{KB}$ kinase (IKK) activates NF- $\kappa B$ via the phosphorylation of the $\mathrm{I} \kappa \mathrm{B}$ on serine-32, leading to the proteolysis of ІкB. TNF $\alpha$ has been shown to promote cellular migration in the tumor microenvironment [26]. As TNF $\alpha$ stimulates the IKK in various cells, we tested the effect of each $\mathrm{EuH}$ extract, including $\mathrm{n}$-hexane, chloroform, ethyl acetate, nbutanol, and aqueous fraction (each $5 \mu \mathrm{g} / \mathrm{mL}$ ), on TNFo-induced IKK phosphorylation in highly metastatic MDA-MB-231 human breast cancer cells. Immunoblot analysis shows that the ethyl acetate fraction of $\mathrm{EuH}(\mathrm{EA} / \mathrm{EuH})$ significantly inhibited TNFo-induced IKK phosphorylation $(P<0.0001$ by Sidak's test) (Fig. 1). We choose the EA/EuH for further investigation of the anti-metastatic activity.

Cell motility is important for tumor cell invasion and metastasis. To determine whether EA/EuH inhibits
TNF $\alpha$-induced migration of highly metastatic MDAMB-231 cells, in vitro scratch wound-healing assay was carried out. After scratching confluent monolayers of MDA-MB-231 cells to create a wound-like gap, the cells were treated with TNFa in the absence or presence of $5 \mu \mathrm{g} / \mathrm{mL} \mathrm{EA} / \mathrm{EuH}$. TNF $\alpha$-treated cells efficiently migrated into the gap area compared to untreated cells (Fig. 2a). However, in the presence of EA/EuH, TNF $\alpha$ induced migration of MDA-MB-231 cells was significantly inhibited (Fig. 2b; $P=0.0006$ by Sidak's test). When MDA-MB-231 cells were treated with $5 \mu \mathrm{g} / \mathrm{mL}$ $\mathrm{EA} / \mathrm{EuH}$ for $8 \mathrm{~h}$, cell viability was not affected by treatment with EA/EuH (Fig. 2c), suggesting that EA/EuH effect on the inhibition of cell migration was not due to its cytotoxicity.

Actin cytoskeletal reorganization plays a key role in the regulation of cellular movement [27]. During cell migration, globular actin monomer (G-actin) assembles to form helical filamentous actin (F-actin)
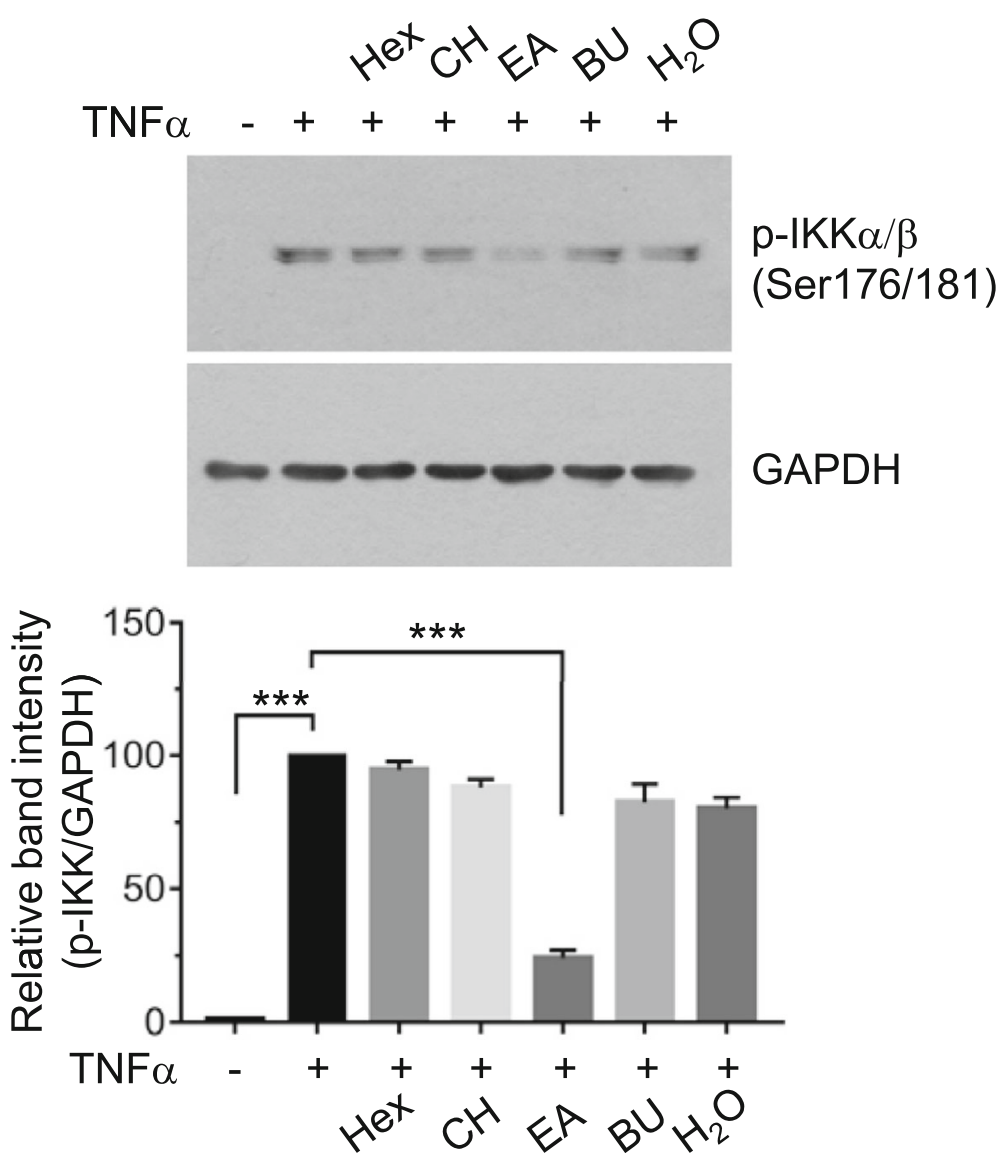

Fig. 1 Effect of E. humifusa Willd (EA/EuH) extracts on TNFa-induced IKK phosphorylation. Serum-starved MDA-MB-231 cells were left untreated or treated with $n$-hexane $(\mathrm{Hex})$, chloroform $(\mathrm{CH})$, ethylacetate $(\mathrm{EA})$, $\mathrm{n}$-butanol $(\mathrm{Bu})$, or aqueous $\left(\mathrm{H}_{2} \mathrm{O}\right)$ fraction (each $\left.5 \mu \mathrm{g} / \mathrm{mL}\right)$ for $30 \mathrm{~min}$ before stimulation with TNFa (10 ng/mL). After $60 \mathrm{~min}$, whole-cell lysates were prepared, and immunoblotting was performed using the phospho-specific antibody against IKKo/ $\beta$ (Ser176/181). The anti-GAPDH antibody was used as an internal control. Band intensities were analyzed using the ImageJ software. TNFa alone treated-band intensities were set to $100 \%$ (control). The relative values of $\%$ of control were plotted as the mean \pm SD. $P$ values were analyzed by Sidak's test. $(n=3)$ 


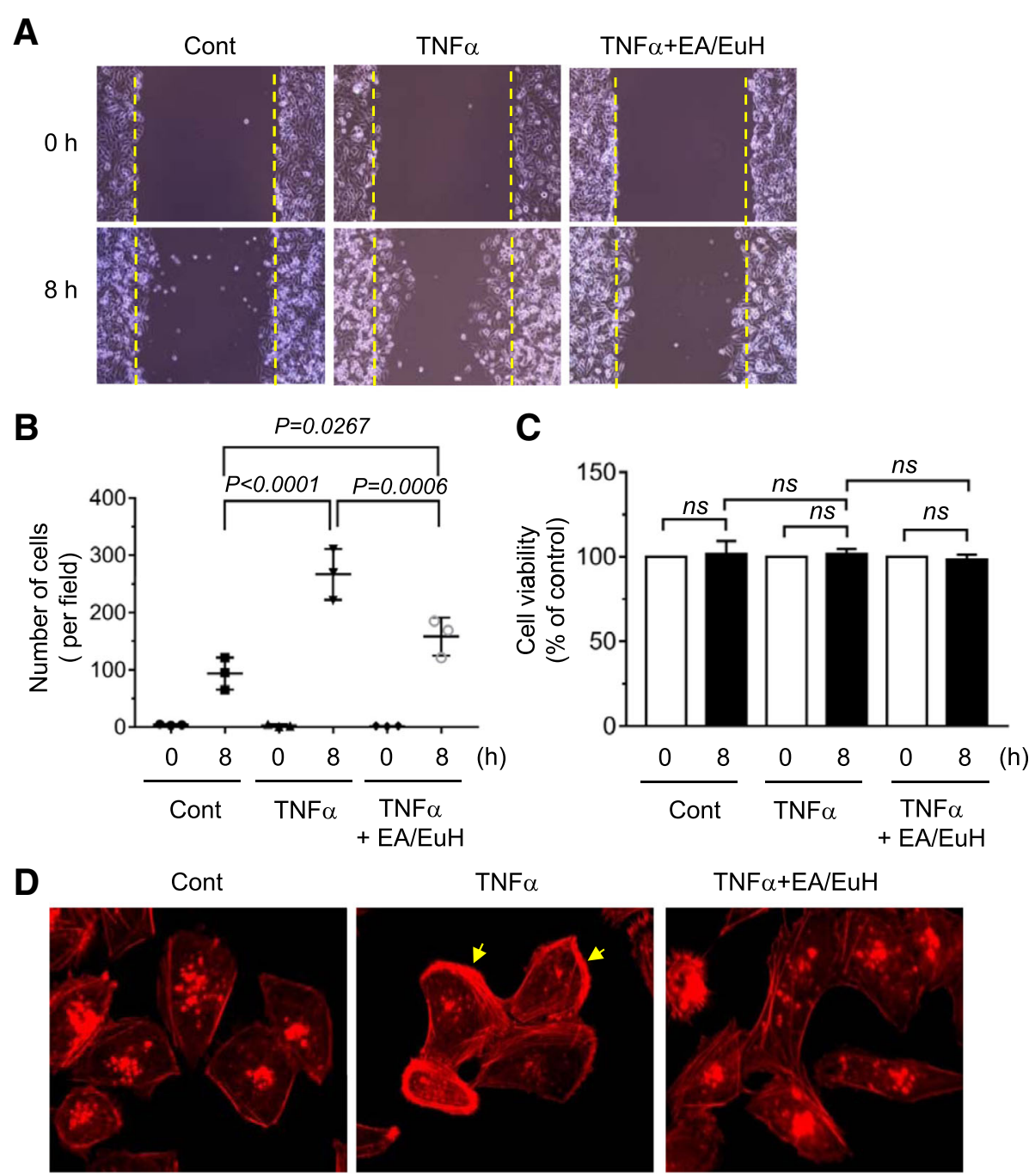

Fig. 2 Effect of ethyl acetate fraction of E. humifusa Willd (EA/EuH) on TNFa-induced motility of MDA-MB-231 cells. a Cell migration assay. MDAMB-231 cells were treated with or without EA/EuH $(5 \mu \mathrm{g} / \mathrm{mL})$ for $30 \mathrm{~min}$, followed by exposure to TNFa $(10 \mathrm{ng} / \mathrm{mL})$. After 8 h, the representative field images were captured by an EVOS FL Auto Cell Imaging System. Dotted lines indicate the scraped boundaries at the beginning of the experiment. $\mathbf{b}$ Cells migrated into the gap area were quantified in a field of view using ImageJ software. The data shown represent the mean \pm SD $(n=3)$. $P$ value was analyzed by Sidak's test. c Cell viability assay. MDA-MB- 231 cells were treated with or without EAVEuH $(5 \mu \mathrm{g} / \mathrm{mL}) \mathrm{for} 30 \mathrm{~min}$, followed by addition of TNFa (10 ng/mL). After $8 \mathrm{~h}$, viable cells were determined using a Cell Counting Kit-8 (CCK-8). The data shown represent the mean \pm SD. $P$ value was analyzed by Sidak's test. $n s$, not-significant. d MDA-MB-231 cells were treated with or without EA/EuH (5 $\mu \mathrm{g} / \mathrm{mL})$ for $30 \mathrm{~min}$, followed by exposure to TNFa (10 ng/mL). After $12 \mathrm{~h}$, the cells were stained with rhodamine-phalloidin (1:100) for $1 \mathrm{~h}$ and actin rearrangement was analyzed. Arrows indicate polarized F-actin

bundles and networks. To determine whether EA/ $\mathrm{EuH}$ affects actin reorganization, F-actin bundle formation was examined by rhodamine-labelled phalloidin, a class of phallotoxins that binds selectively to F-actin. MDA-MB-231 cells treated with $10 \mathrm{ng} / \mathrm{mL}$ TNF $\alpha$ displayed a polarized pattern of Factin distributed mostly at cell edge, which was substantially reduced by $5 \mu \mathrm{g} / \mathrm{mL}$ EA/EuH (Fig. 2d). These data demonstrate that EA/EuH attenuates TNF $\alpha$-induced migratory activity of MDA-MB-231 cells.

\section{EA/EuH inhibits TNFa-induced invasive capability of} MDA-MB-231 breast cancer cells

In addition to cell migration, invasiveness into the surrounding ECM is a critical feature of metastatic cancer cells. To evaluate the inhibitory effect of $\mathrm{EA} / \mathrm{EuH}$ on tumor invasion, 3-D spheroids of MDA-MB-231 cells were formed in an ECM-like environment and then monitored for their invasive capability. As shown in Fig. 3a, the MDA-MB-231 spheroids remained noninvasive under unstimulated conditions. After 3 days of $10 \mathrm{ng} / \mathrm{mL}$ TNFa stimulation, the cells began to spread 
A

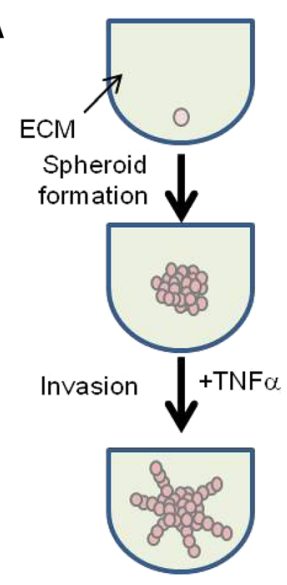

B

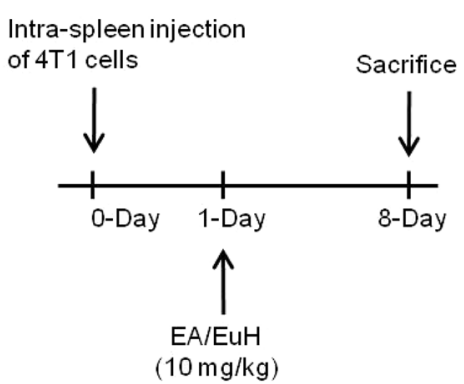

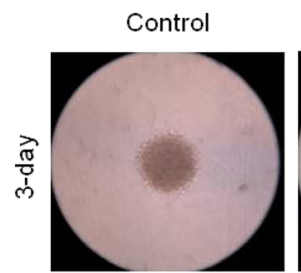

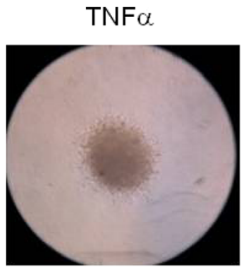

TNF $\alpha+$ EA/EuH
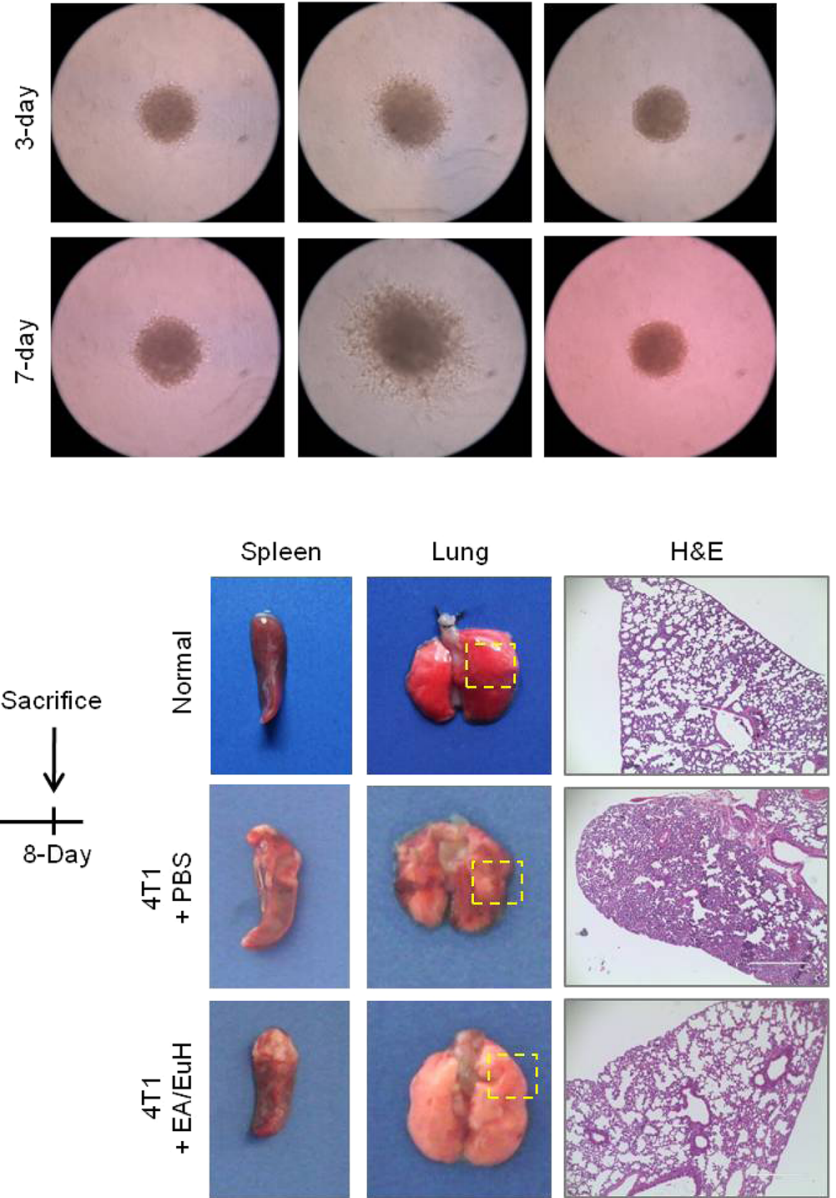

Fig. 3 Inhibitory effect of EA/EuH on the invasive capability of MDA-MB-231 cells and experimental lung metastasis of 4 T1 cells. a MDA-MB-231 cell spheroids in the extracellular matrix were either untreated (control) or treated with TNFa $(10 \mathrm{ng} / \mathrm{mL}$ ) in the absence or presence of EA/EuH $(5 \mathrm{\mu g} / \mathrm{mL})$. Morphology of 3-D cell invasion was captured at 3-days and 7-days with an EVOS FL Auto Cell Imaging System. b Mouse 4 T1 mammary tumor cells were implanted into the spleen of Balb/c mouse. At 1 day post intra-splenic implantation of 4 T1 cells, either phosphate buffered saline (PBS) or EA/EuH (10 mg/kg) was administered intraperitoneally daily. After 8 days, mice were sacrificed. Lung tissues were fixed and stained with hematoxylin and eosin (H\&E)

out of the spheroid into the surrounding matrix with a typical starburst pattern. However, in the presence of $5 \mu \mathrm{g} / \mathrm{mL} \mathrm{EA} / \mathrm{EuH}$, TNF $\alpha$-induced invasive protrusion was almost absent. These data suggest that EA/EuH could inhibit the invasive capability of breast cancer cells.

\section{EA/EuH reduces experimental lung metastasis of $4 \mathrm{~T} 1$ mouse mammary tumor cells in vivo}

We next investigated whether $\mathrm{EA} / \mathrm{EuH}$ inhibits tumor metastasis in vivo. Since tumor metastatic progression is strongly influenced by host immune responses and the interaction of tumor cells with tumor microenvironment, we employed a mouse syngeneic tumor metastasis model. 4 T1 mouse mammary carcinoma cells are highly invasive and can metastasize to multiple sites, including the lung [28]. We implanted $4 \mathrm{~T} 1$ cells into the spleen of Balb/c mouse $(n=15)$, and they were allowed to form tumors. After 1 day, we randomized the mice into two groups, with one control group receiving phosphate buffered saline (PBS) $(n=5)$ and the other receiving $10 \mathrm{mg} / \mathrm{kg} \mathrm{EA} / \mathrm{EuH}(n=7)$ daily by intraperitoneal injection. Eight days after implantation, all mice were sacrificed. As shown in Fig. 3b, mice that received EA/EuH showed smaller metastatic foci in the lungs as compared to control group mice. These data suggest that EA/EuH has a potent inhibitory effect against experimental lung metastasis.

\section{EA/EuH suppresses TNFa-induced MMP-9 mRNA expression}

For migration and invasion of tumor cells, proteolytic disruption of basement membranes and ECM proteins is 
critical [3]. MMP-9 degrades collagen in the basement membrane and ECM. MMP-9 levels are closely linked to the promotion of tumor invasion and metastasis, and specific inhibitors of MMP have been shown to inhibit tumor cell invasion [29]. Indeed, MMP-9 is overexpressed in breast cancers and is associated with the promotion of metastasis [30].

Given that TNF $\alpha$ induces MMP-9 expression in many types of cancer [31, 32], we wondered whether EA/EuH inhibits TNF $\alpha$-induced expression of MMP-9 in various breast cancer cells. RT-PCR analysis showed that treatment with $10 \mathrm{ng} / \mathrm{mL}$ TNFo upregulated MMP-9 mRNA expression in MDA-MB-231, MDA-MB-435, T47D, and MCF7 human breast cancer cells, while this induction was substantially abolished in the presence of $5 \mu \mathrm{g} / \mathrm{mL}$ EA/EuH (Fig. 4a). To precisely quantify the changes in MMP-9 mRNA expression, quantitative real-time PCR analysis was performed (Fig. 4b). Treatment with $10 \mathrm{ng} /$ $\mathrm{mL}$ TNF $\alpha$ alone resulted in $330 \pm 65.6-, 313 \pm 35.1-, 186 \pm$ 61.2, and $233 \pm 56.9$-fold increases in MMP-9 mRNA levels in MDA-MB-231, MDA-MB-435, T47D, and MCF7 cells, respectively. However, these were significantly decreased to $75.7 \pm 27.2-, \quad 35.0 \pm 8.89-, \quad 16.3 \pm 7.57$-, and $22.7 \pm 9.71$-fold increases upon pre-treatment with $5 \mu \mathrm{g} / \mathrm{mL} \mathrm{EA} / \mathrm{EuH}$, respectively (all $P<0.01$ by Sidak's test). These data suggest that $\mathrm{EA} / \mathrm{EuH}$ can downregulate TNF $\alpha$-induced MMP-9 mRNA expression in various breast cancer cells.

\section{EA/EuH inhibits TNFa-induced MMP-9 expression at the promoter level}

To understand the molecular mechanism underlying EA/EuH-induced downregulation of MMP-9 mRNA expression, we examined the effect of EA/EuH on
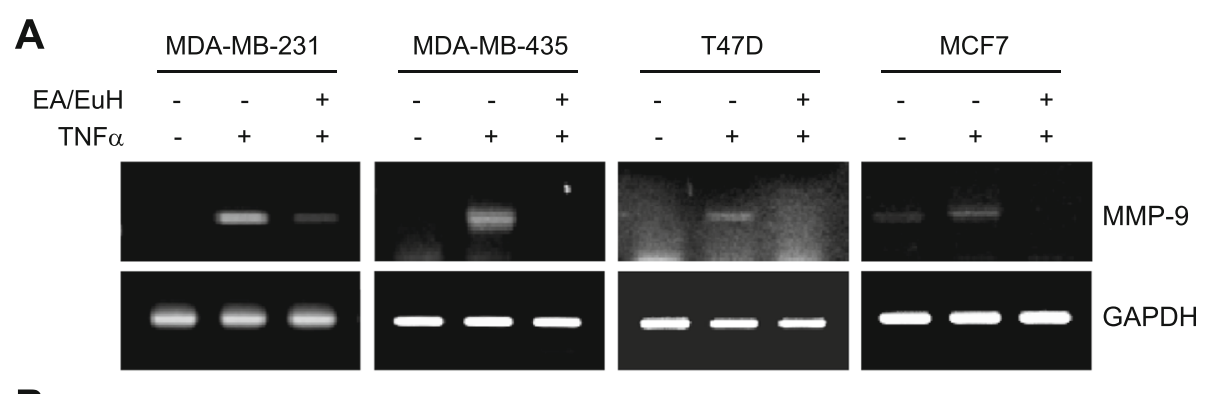

B
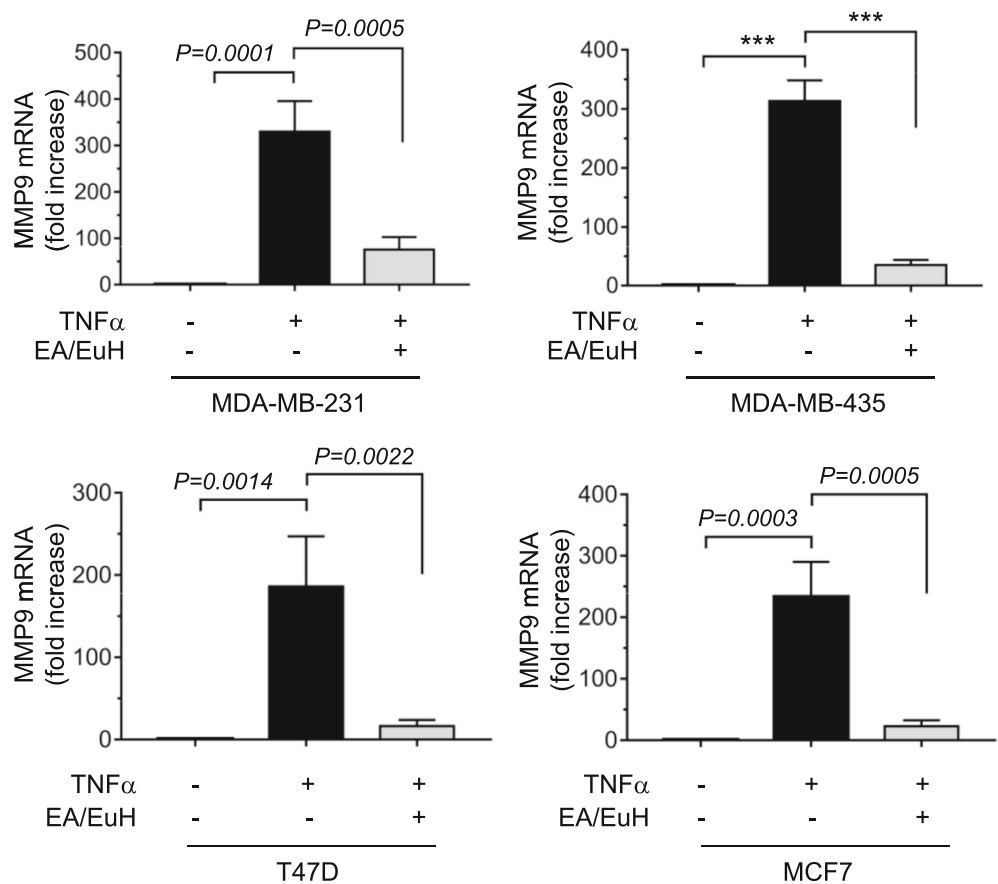

Fig. 4 Inhibitory effect of EA/EuH on TNFa-induced MMP-9 mRNA expression. a RT-PCR. MDA-MB-231, MDA-MB-435, T47D, and MCF7 cells were treated with or without TNFa $(10 \mathrm{ng} / \mathrm{mL})$ in the absence of presence of EA/EuH $(5 \mu \mathrm{g} / \mathrm{mL})$ for $18 \mathrm{~h}$. Total RNA was isolated and then RT-PCR was performed. GAPDH mRNA was used as an internal control. b Real-time PCR. MDA-MB-231, MDA-MB-435, T47D, and MCF7 cells were treated as in (a). MMP-9 mRNA levels were measured by quantitative real-time PCR. The relative fold changes were normalized to GAPDH mRNA in the same sample. The data shown represent the mean $\pm \mathrm{SD}(n=3) .{ }^{* * *}, P<0.0001$. $P$ value was analyzed by Sidak's test 
A

pMMP9-Luc(-925/+13)

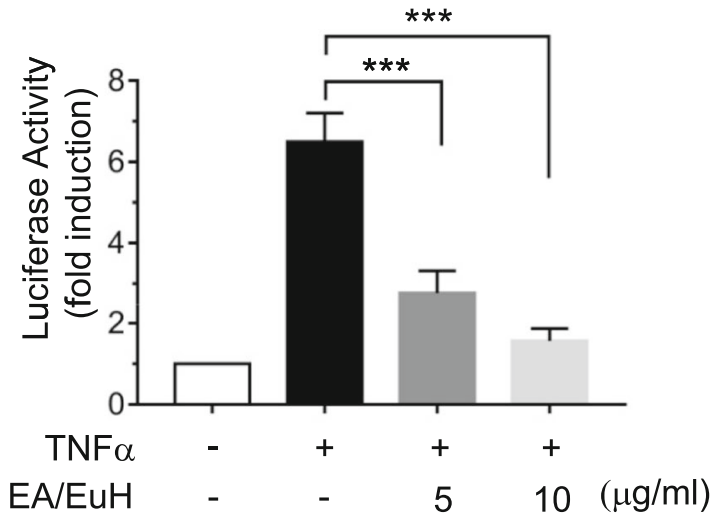

$\mathbf{B}$
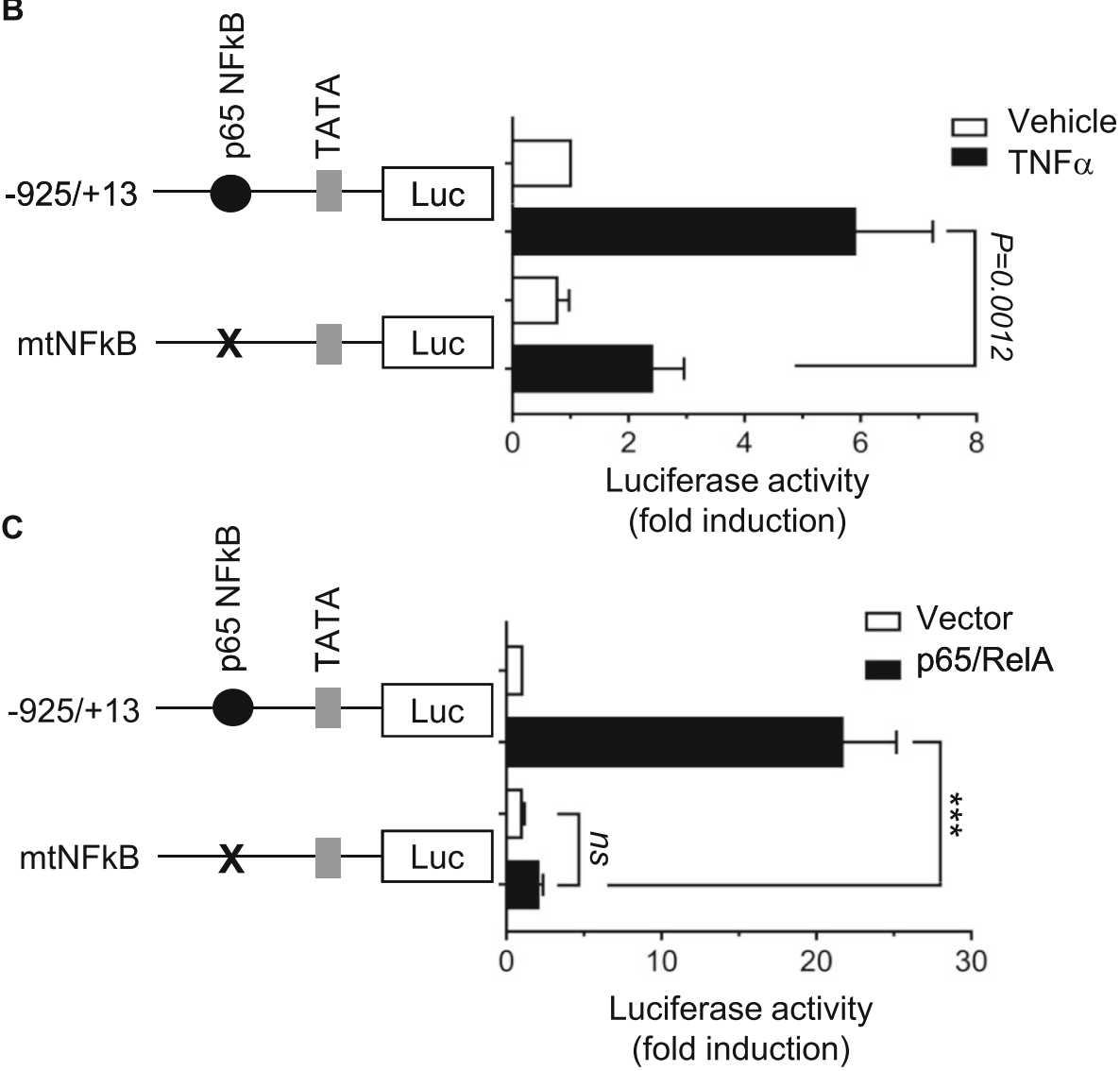

Fig. 5 Inhibitory effect of EA/EuH on TNFa-induced MMP-9 promoter activation. a Promoter assay. MDA-MB-231 cells were transfected with $0.2 \mu \mathrm{g}$ of promoter reporter, pMMP9-Luc(-925/+13). After $48 \mathrm{~h}$, cells were treated with Ea/EuH $(5 \mu \mathrm{g} / \mathrm{mL})$ for $8 \mathrm{~h}$, and their luciferase activities were measured. Firefly luciferase activity was normalized to Renilla luciferase activity. The data shown represent the mean \pm SD $(n=9)$. ***, $P<0.0001$. $P$ value was analyzed by Sidak's multiple comparisons test. b Either pMMP9-Luc(-925/+13) or pMMP9-LuC(-925/+13)mtNFKB was transfected into MDA-MB-231 cells. After $48 \mathrm{~h}$, cells were treated with TNFa $(10 \mathrm{ng} / \mathrm{mL})$ for $8 \mathrm{~h}$, and their luciferase activities were measured. The data shown represent the mean \pm SD $(n=9)$. $P$ value was analyzed by Sidak's test. c Either pMMP9-Luc(-925/+13) or pMMP9-Luc(-925/+13)mtNFkB was co-transfected along with RelA/p65 nuclear factor kappa B (NF-kB) expression plasmid into MDA-MB-231 cells. After 48 h, cells were collected, and their luciferase activities were measured. The data shown represent the mean \pm SD $(n=9)$. $n s$, not significant. ${ }^{* *}, P<0.0001$. $P$ value was analyzed by Sidak's multiple comparisons test 
MMP-9 gene promoter activity. MDA-MB-231 cells were transfected with MMP-9 promoter reporter, pMMP9-Luc $(-925 /+13)$, and the luciferase reporter activity was assessed. We found that TNF $\alpha$-induced MMP-9 promoter activity was significantly inhibited in a dose-dependent manner by $5 \mu \mathrm{g} / \mathrm{mL} \mathrm{EA} / \mathrm{EuH}$ treatment (all $P<0.0001$ by Sidak's test; Fig. 5a), indicating that $\mathrm{EA} / \mathrm{EuH}$ reduced MMP-9 expression at the transcriptional level. The MMP-9 gene is regulated by multiple regulatory proteins, including NF $-\kappa B$ [32]. We also found that disruption of the NF-кB-binding site by site-directed mutagenesis abrogated TNF $\alpha$-induced MMP-9 gene promoter activity (Fig. 5b) and RelA/p65 NF-kB-induced MMP-9 gene promoter activity (Fig. $5 \mathrm{c}$ ). Thus, it is possible that EA/EuH-induced downregulation of MMP-9 expression is associated with the inhibition of NF- $\mathrm{kB}$ transcriptional activity.

\section{EA/EuH attenuates TNFa-induced NF-kB activation}

To investigate whether EA/EuH could inhibit NF- $\mathrm{B}$, MDA-MB-231 cells were treated with $10 \mathrm{ng} / \mathrm{mL} \mathrm{TNF} \alpha$ for $20 \mathrm{~min}$ in the absence or presence of EA/EuH. Phosphorylation status of $\mathrm{I} \kappa \mathrm{B}$ and RelA/p65 NF- $\mathrm{kB}$ was analyzed by immunoblot analysis. Treatment with EA/EuH dose-dependently inhibited TNF $\alpha$-induced phosphorylation of IKB on serine-32 and RelA/p65 on serine-536 (Fig. 6a), suggesting that EA/EuH blocked the signaling pathway regulating I $\mathrm{B}$ upstream kinase. To address further the inhibitory effect of EA/EuH on NF- $\mathrm{kB}$, RelA/ p65 phosphorylation was analyzed in MDA-MB-231 cells using immunofluorescent microscopy. Fluorescent
A

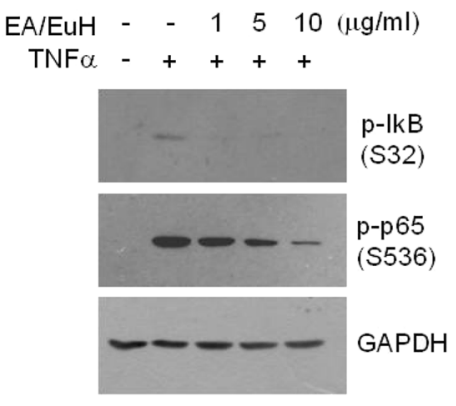

B

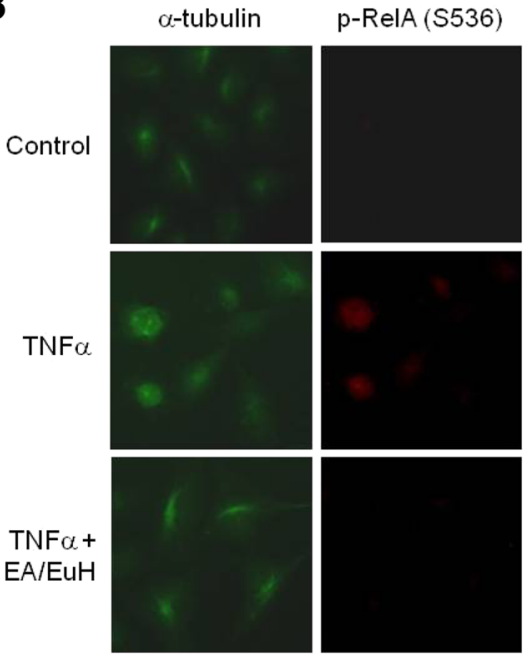

C
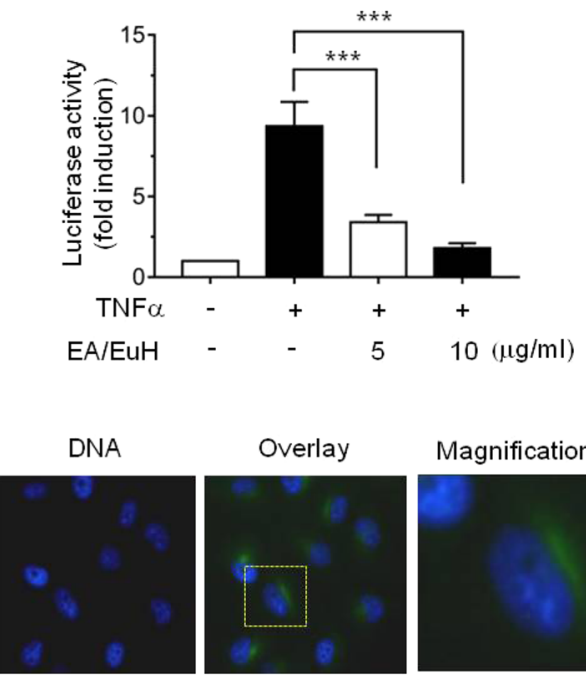

Magnification
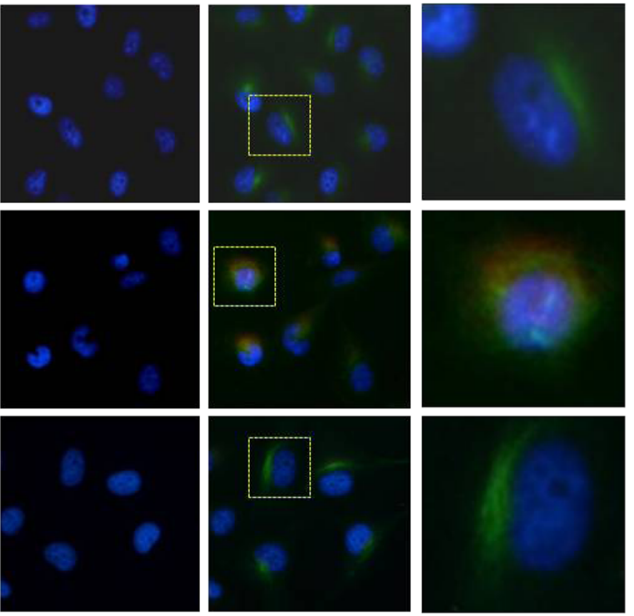

Fig. 6 Inhibitory effect of EA/EuH on TNFa-induced NF-KB activation. a Serum-starved MDA-MB-231 cells were pre-treated with EA/EuH (5 $\mu$ g/mL) for 30 min before stimulation with TNFa (10 ng/mL). After 20 min, whole-cell lysates were prepared, and immunoblotting was performed using the phospho-specific antibody against IkBa (Ser32) or RelA/p65 (Ser536) as indicated. The anti-GAPDH antibody was used as an internal control. b MDA-MB-231 cells were pre-treated with EAVEuH $(5 \mu \mathrm{g} / \mathrm{mL})$ for $30 \mathrm{~min}$ before stimulation with TNFa (10 ng/mL). After $20 \mathrm{~min}$, the cells were fixed and incubated with antibodies against a-tubulin or phospho-RelA/p65 (Ser536) for 2 h, followed by incubation with Alexa Fluor 488-conjugated (green signal) or Alexa Fluor 555-conjugated (red signal) secondary antibody for 30 min. Nuclear DNA was stained with 1 mg/mL Hoechst 33258 for 10 min (blue signal). c MDA-MB-231 cells were transfected with $5 \times$ NFkB-Luc plasmid along with 50 ng pRL-null. At 48 h post-transfection, the cells were treated with or without $10 \mathrm{ng} / \mathrm{mL}$ TNFa in the absence or presence of EAVEuH $(5 \mu \mathrm{g} / \mathrm{mL})$. The data shown represent the mean \pm SD. ${ }^{* * *}, P<0.0001$ $(n=9)$. $P$ value was analyzed by Sidak's multiple comparisons test 
staining for phospho-RelA at serine-536 was evident in the perinucleus and nucleus upon TNF $\alpha$ stimulation, which was suppressed by EA/EuH treatment (Fig. 6b). We next wondered whether EA/EuH-induced inhibition of NF-kB phosphorylation is functionally linked to the inhibition of transcriptional activity. NF-kB-dependent transcription was measured using a NF- $\mathrm{kB}$ cis-acting luciferase report system. Upon $10 \mathrm{ng} / \mathrm{mL}$ TNF $\alpha$ stimulation, NF- $\mathrm{kB}$ transcriptional activity was increased $9.3 \pm$ 1.5 -fold; however, this increase in transcriptional activity was significantly decreased in the presence of $5 \mu \mathrm{g} / \mathrm{mL}$ EA/EuH (all $P<0.0001$ by Sidak's test; Fig. 6c), suggesting that $\mathrm{EA} / \mathrm{EuH}$ inhibits NF-kB-regulated gene transcription.

\section{Conclusion}

E. humifusa Willd has been used in traditional medicine in the eastern Asia, including Korea and China, for a long time. This study demonstrates that the ethyl acetate fraction from $E$. humifusa Willd $(\mathrm{EA} / \mathrm{EuH})$ has a preventive effect on TNF $\alpha$-induced invasive capability of highly metastatic MDA-MB-231 breast cancer cells through the inhibition of NF-kB-mediated MMP-9 gene expression. We also show that EA/EuH attenuated experimental lung metastasis in vivo as revealed by syngenic intra-splenic transplantation model of 4 T1 mouse mammary carcinoma cells. These findings suggest that E. humifusa Willd may be beneficial in the prevention of invasion and metastasis of early stage breast cancer.

\section{Abbreviations}

EA: Ethyl acetate; ECM: Extracellular matrix; EuH: Euphorbia humifusa Willd; GAPDH: Glyceraldehyde-3-phosphate dehydrogenase; H\&E: Hematoxylin and eosin; MMP: Matrix metalloproteinase; TNFa: Tumour necrosis factor alpha

\section{Acknowledgements}

This paper was supported by the KU Research Professor Program of Konkuk University.

\section{Funding}

This study was supported by the Agenda program (RDA, PJ010484) from the Rural Development Administration and by a grant from the Priority Research Centers Program through the National Research Foundation of Korea funded by the Ministry of Education, Science and Technology (NRF-2009-0093824).

\section{Availability of data and materials}

The datasets supporting the conclusions of this article are presented in this main paper.

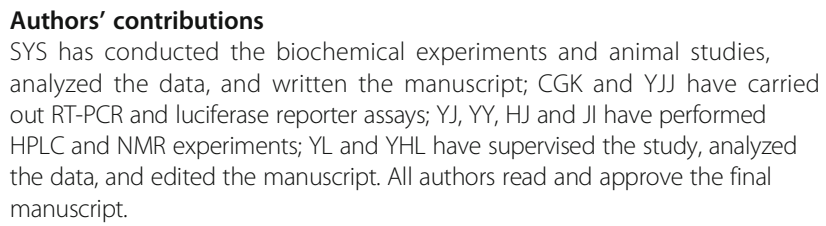

Authors' contributions

SYS has conducted the biochemical experiments and animal studies, analyzed the data, and written the manuscript; CGK and YJJ have carried out RT-PCR and luciferase reporter assays; YJ, YY, HJ and Jl have performed HPLC and NMR experiments; YL and YHL have supervised the study, analyzed the data, and edited the manuscript. All authors read and approve the final manuscript.

\section{Competing interests}

The authors declare that they have no competing interests.

\section{Consent for publication}

Not applicable.

\section{Ethics approval and consent to participate}

Present work was approved by the Ethics Committee at the Konkuk University, and all experiments were performed in accordance with the guidelines of the National Animal Care and Use Committee.

\section{Author details}

${ }^{1}$ Department of Biological Sciences, College of Biological Science and Biotechnology, Konkuk University, Seoul, Republic of Korea. ${ }^{2}$ Cancer and Metabolism Institute, Konkuk University, Seoul, Republic of Korea. ${ }^{3}$ Division of Bioscience and Biotechnology, College of Biological Science and Biotechnology, BMIC, Konkuk University, Seoul, Republic of Korea. ${ }^{4}$ College of Biological Science and Biotechnology, Konkuk University, 120 Neundong-ro, Gwangjin-gu, Seoul 05029, Republic of Korea.

Received: 20 June 2016 Accepted: 11 October 2016

Published online: 24 October 2016

\section{References}

1. Jemal A, Bray F, Center MM, Ferlay J, Ward E, Forman D. Global cancer statistics. CA Cancer J Clin. 2011;61(2):69-90

2. Balkwill F. Tumour necrosis factor and cancer. Nat Rev Cancer. 2009;9(5): 361-71.

3. Deryugina El, Quigley JP. Matrix metalloproteinases and tumor metastasis. Cancer Metastasis Rev. 2006;25(1):9-34.

4. Orlowski RZ, Baldwin Jr AS. NF-kappaB as a therapeutic target in cancer. Trends Mol Med. 2002:8(8):385-9.

5. Nabel GJ, Verma IM. Proposed NF-kappa B/I kappa B family nomenclature. Genes Dev. 1993;7(11):2063.

6. Aggarwal BB. Nuclear factor-kappaB: the enemy within. Cancer Cell. 2004; 6(3):203-8.

7. Sovak MA, Bellas RE, Kim DW, Zanieski GJ, Rogers AE, Traish AM, Sonenshein GE. Aberrant nuclear factor-kappaB/Rel expression and the pathogenesis of breast cancer. J Clin Invest. 1997:100(12):2952-60.

8. Bond M, Fabunmi RP, Baker AH, Newby AC. Synergistic upregulation of metalloproteinase- 9 by growth factors and inflammatory cytokines: an absolute requirement for transcription factor NF-kappa B. FEBS Lett. 1998; 435(1):29-34.

9. Keifer JA, Guttridge DC, Ashburner BP, Baldwin Jr AS. Inhibition of NF-kappa $B$ activity by thalidomide through suppression of IkappaB kinase activity. J Biol Chem. 2001;276(25):22382-7.

10. Hideshima T, Chauhan D, Richardson P, Mitsiades C, Mitsiades N, Hayashi T, Munshi N, Dang L, Castro A, Palombella V, et al. NF-kappa B as a therapeutic target in multiple myeloma. J Biol Chem. 2002;277(19):16639-47.

11. Fabre C, Mimura N, Bobb K, Kong SY, Gorgun G, Cirstea D, Hu Y, Minami J, Ohguchi H, Zhang J, et al. Dual inhibition of canonical and noncanonical NF-kappaB pathways demonstrates significant antitumor activities in multiple myeloma. Clin Cancer Res. 2012;18(17):4669-81.

12. Wang $\Pi$, Zhou GH, Kho JH, Sun YY, Wen JF, Kang DG, Lee HS, Cho KW, Jin SN. Vasorelaxant action of an ethylacetate fraction of Euphorbia humifusa involves NO-cGMP pathway and potassium channels. J Ethnopharmacol. 2013;148(2):655-63.

13. Tian $Y$, Sun LM, Liu XQ, Li B, Wang Q, Dong JX. Anti-HBV active flavone glucosides from Euphorbia humifusa Willd. Fitoterapia. 2010;81(7):799-802.

14. Deng F, Tang N, Xu J, Shi YH, Zhao M, Zhang JS. New alpha-pyrrolidinonoids and glycosides from Euphorbia humifusa. J Asian Nat Prod Res. 2008;10(5-6): 531-9.

15. Yoshida T, Amakura Y, Liu YZ, Okuda T. Tannins and related polyphenols of euphorbiaceous plants. XI. Three new hydrolyzable tannins and a polyphenol glucoside from Euphorbia humifusa. Chem Pharm Bull (Tokyo). 1994;42(9):1803-7.

16. Luyen BT, Tai BH, Thao NP, Eun KJ, Cha JY, Xin MJ, Lee YM, Kim YH. Anti-inflammatory components of Euphorbia humifusa Willd. Bioorg Med Chem Lett. 2014;24(8):1895-900.

17. Li ZJ, Guo X, Dawuti G, Aibai S. Antifungal activity of ellagic acid in vitro and in vivo. Phytother Res. 2015;29(7):1019-25.

18. Tian Y, Sun LM, Li B, Liu XQ, Dong JX. New anti-HBV caryophyllane-type sesquiterpenoids from Euphorbia humifusa Willd. Fitoterapia. 2011;82(2):251-4.

19. Shin SY, Nam JS, Lim Y, Lee YH. TNFalpha-exposed bone marrow-derived mesenchymal stem cells promote locomotion of MDA-MB-231 breast cancer cells through transcriptional activation of CXCR3 ligand chemokines. J Biol Chem. 2010;285(40):30731-40. 
20. Lee MS, Koh D, Kim GS, Lee SE, Noh HJ, Kim SY, Lee YH, Lim Y, Shin SY. 2-Hydroxy-3,4-naphthochalcone (2H-NC) inhibits TNFalpha-induced tumor invasion through the downregulation of NF-kappaB-mediated MMP-9 gene expression. Bioorg Med Chem Lett. 2015;25(1):128-32.

21. Giavazzi R, Jessup JM, Campbell DE, Walker SM, Fidler IJ. Experimental nude mouse model of human colorectal cancer liver metastases. J Natl Cancer Inst. 1986;77(6):1303-8.

22. Shin SY, Lee JM, Lim Y, Lee YH. Transcriptional regulation of the growthregulated oncogene alpha gene by early growth response protein-1 in response to tumor necrosis factor alpha stimulation. Biochim Biophys Acta. 2013;1829(10):1066-74.

23. Shin SY, Kim JH, Yoon H, Choi YK, Koh D, Lim Y, Lee YH. Novel antimitotic activity of 2-hydroxy-4-methoxy-2,'3'-benzochalcone (HymnPro) through the inhibition of tubulin polymerization. J Agric Food Chem. 2013; 61(51):12588-97.

24. Shin SY, Kim JH, Baker A, Lim Y, Lee YH. Transcription factor Egr-1 is essential for maximal matrix metalloproteinase-9 transcription by tumor necrosis factor alpha. Mol Cancer Res. 2010;8(4):507-19.

25. Van den Steen PE, Dubois B, Nelissen I, Rudd PM, Dwek RA, Opdenakker G. Biochemistry and molecular biology of gelatinase $B$ or matrix metalloproteinase-9 (MMP-9). Crit Rev Biochem Mol Biol. 2002;37(6):375-536.

26. Mantovani A, Allavena P, Sica A, Balkwill F. Cancer-related inflammation. Nature. 2008:454(7203):436-44.

27. Fletcher DA, Mullins RD. Cell mechanics and the cytoskeleton. Nature. 2010; 463(7280):485-92.

28. Aslakson CJ, Miller FR. Selective events in the metastatic process defined by analysis of the sequential dissemination of subpopulations of a mouse mammary tumor. Cancer Res. 1992;52(6):1399-405.

29. Ura H, Bonfil RD, Reich R, Reddel R, Pfeifer A, Harris CC, Klein-Szanto AJ. Expression of type IV collagenase and procollagen genes and its correlation with the tumorigenic, invasive, and metastatic abilities of oncogenetransformed human bronchial epithelial cells. Cancer Res. 1989;49(16): 4615-21.

30. Scorilas A, Karameris A, Arnogiannaki N, Ardavanis A, Bassilopoulos P, Trangas T, Talieri M. Overexpression of matrix-metalloproteinase-9 in human breast cancer: a potential favourable indicator in node-negative patients. Br J Cancer. 2001:84(11):1488-96.

31. Joyce JA, Pollard JW. Microenvironmental regulation of metastasis. Nat Rev Cancer. 2009;9(4):239-52.

32. Miriam F-F, Alicia R. Folgueras, Sandra C, Carlos L-O. Matrix metalloproteinases: Evolution, gene regulation and functional analysis in mouse models. Biochim Biophys Actal. 2010;1803(1):3-19.

\section{Submit your next manuscript to BioMed Central and we will help you at every step:}

- We accept pre-submission inquiries

- Our selector tool helps you to find the most relevant journal

- We provide round the clock customer support

- Convenient online submission

- Thorough peer review

- Inclusion in PubMed and all major indexing services

- Maximum visibility for your research

Submit your manuscript at www.biomedcentral.com/submit 